Morón-Marchena, J.A. (2014). Educación y personas mayores. Revista Electrónica Interuniversitaria de Formación del Profesorado, 17 (1), 107-121.

DOI: http://dx.doi.org/10.6018/reifop.17.1.198871

\title{
Educación y personas mayores
}

\author{
Juan Agustín Morón Marchena \\ Universidad Pablo de Olavide (Sevilla)
}

\section{Resumen}

Al realizar una proyección de población española, se demuestra que cada vez habrá más personas mayores. Tanto en números globales, en el número total, como especialmente en el porcentaje sobre el total de la población. Será una sociedad en progresivo envejecimiento. Pero se vivirá más tiempo, porque aumentará significativamente la esperanza de vida, con mejores condiciones de salud. Y, por tanto, habrá más tiempo para, por ejemplo, formarse, asistir a clases... El aprendizaje a lo largo de toda la vida ganará cada vez más espacio. La formación del profesorado (la formación de los educadores y formadores) tiene en las Personas Mayores un campo de acción muy importante y en crecimiento. Una formación con un carácter específico dentro de la Educación Permanente y de la Educación de Personas Adultas, que requiere un enfoque

\section{Palabras clave}

Mayores; Educación a lo largo de toda la vida; Educación de Personas Adultas; Formación docente.

\section{Education and elderly people}

\section{Abstract}

When making a projection of Spanish population, it is shown that there will be more and more elderly people. In overall numbers as well as in total numbers, and especially in the 
percentage on the whole population. It will be a society in an increasing aging. But people will live longer, as life expectancy will increase significantly with better health conditions. Hence there will be more time, for example, for training, to attend classes... . Life Long Learning will gain more and more space. Teachers' training (Training of Educators and Teachers) has in elderly people, a really important and growing action field. A training with a specific character within Life Long Learning and Adult Education, which requires a different approach to the conceptual, as well as procedural and methodolocical planning

\title{
Key words
}

Elderly people; Life Long Learning; Adult education; Teacher education.

\section{Introducción}

\begin{abstract}
"Algún lector podría sorprenderse que se aborde monográficamente asuntos relativos al envejecimiento, sus repercusiones y posibilidades en este tiempo. ¿Qué tenemos que ver con los mayores? ¿Acaso no es más que suficiente con atender a los procesos de educación formal? ¿No son acaso los niños/as y jóvenes los destinatarios por antonomasia de la acción educativa y, por tanto, también de la formación de profesores que de ellos se encargan?...".
\end{abstract}

Así comenzaba el Editorial 'Retos Educativos del Envejecimiento', como presentación del Tema Monográfico sobre 'Perfiles del Envejecimiento' en la Revista Interuniversitaria de Formación del Profesorado (2002, $\left.n^{\circ} 45\right)$. Han pasado algunos años, pero esos interrogantes mantienen su actualidad intacta: me temo que esas mismas preguntas se siguen haciendo hoy en día.

La Formación del Profesorado tiene ya un largo recorrido temporal. Y su campo de acción también es amplio, abarcando distintos ámbitos de diversa naturaleza. Unos con una dilatada tradición y trayectoria histórica; otros más novedosos en el tiempo, de rotunda actualidad e interés; algunos que resurgen 'guadianescamente' (permítanme el vocablo) cada cierto tiempo... En los manuales de referencia de Formación del Profesorado o en la propia Revista Interuniversitaria de Formación del Profesorado podemos conocer el estado de la cuestión. Uno de los temas menos tratado es el referido a la Formación del Profesorado y las Personas Mayores. Ciertamente, al día de hoy, podemos afirmar que los más mayores no se constituyen apenas en objeto de estudio para la disciplina.

Hay mucho escrito e investigado sobre la Formación de Profesorado en las etapas de Infantil, Primaria, Secundaria, Formación Profesional, Educación Superior, Universidad, etc. Pero poco, muy poco, sobre Formación del Profesorado y Personas Mayores.

Sin embargo, un bloque de población tan amplio y numeroso debería ser motivo de ocupación para cualquier ciencia. De la misma manera que la educación, que la perspectiva educativa, en general, tiene una preocupación cada vez mayor por las personas más mayores (y viceversa, diría), todas las disciplinas o materias que conforman aquella deberían asimismo poner su vista en las personas mayores.

Fundamentación teórica, epistemología, competencias, metodologías y procedimientos, planteamientos didácticos, técnicas y herramientas... todos aquellos aspectos que se tratan en cualquier etapa educativa o sobre cualquier tipo de educandos, también deberían ser objetos de estudio, y de propuestas, en el caso de las Personas Mayores. 
En este sentido, y creemos que oportunamente, cada vez se aborda más en encuentros y publicaciones científicos. Valga como ejemplo el reciente Congreso Internacional de Formación de Profesorado incluye entre las Áreas Temáticas una específica sobre 'Educación y Personas Mayores'. O la propia revista RIFOP -y REIFOP-, con artículos diversos.

A ello dedicaremos estas líneas, a reflexionar sobre la pertinencia de que la Formación del Profesorado tenga entre sus campo de acción a las Personas Mayores. Diría más, justificar porqué debe ser uno de sus elementos de atención. De la misma manera que somos conscientes que los más mayores tienen en la educación y formación uno de sus intereses, también la educación y la formación tienen en las personas mayores un colectivo que atender.

\section{Un primer argumento: una cuestión de números}

La población de España es de 46.704.314 habitantes (cifra oficial del Instituto Nacional de Estadística, tomando como base el último Censo de Población oficial, a 1 de enero de 2013). En el último año se ha producido un descenso del 0,2\% de la población, la primera vez que disminuye desde que se disponen de datos anuales (las series datan desde 1971). Aunque hay un saldo vegetativo favorable de 48.488 personas (es decir, más nacimientos que defunciones), no compensa el saldo migratorio negativo, de 162.390 personas (por la disminución de los inmigrantes y por el aumento de emigrantes al exterior).

Este descenso de población afecta principalmente al grupo de edad entre los 15 y 39 años y entre los menores de 10 años. Sin embargo, hay un incremento entre los más mayores: la población de más de 64 años ha aumentado un 1,7\% en el último año (algo más de 134.000 personas). Este incremento se produce en todas las edades de más de 65 años, excepto entre los de 75 a 79 años, que sí disminuyen (el motivo se debe a que es la generación nacida durante la Guerra Civil).

Los más mayores no son los grupos de edad protagonista en los procesos migratorios, ni en inmigración ni en los movimientos de emigración, que sí afectan mucho más a otros grupos de edad.

Estos son los datos actuales. Pero, ¿qué ocurrirá en los próximos años? Pues que se producirá un envejecimiento de la población española, fundamentalmente por el descenso de la natalidad y por el saldo migratorio negativo.

Según el reciente estudio del Instituto Nacional de Estadística sobre la proyección de la población española a corto plazo, es decir, para los próximos 10 años, si se mantienen las tendencias demográficas actuales, nuestro país perderá el 5,6\% de la población, que será de algo más de 44 millones de habitantes en el 2023 (INE, 2013).

Este descenso se debería a la disminución de los nacimientos (que se viene produciendo desde el 2009), de tal manera que dentro de 10 años habría un $25 \%$ menos de nacimientos que ahora, a pesar una ligera tendencia favorable a la fecundidad de las mujeres ( 1,41 frente al 1,34 actual). Además de posibles factores sociales, este hecho se debe también a la reducción del número de mujeres en edad fértil por la crisis de natalidad de finales de los años ochenta y de los noventa.

Habrá una disminución significativa en los tramos de población de entre los 20 y 50 años de edad (reducción del $22,7 \%$ con respecto a la actualidad, unos 4,7 millones de personas) y de los menores de 10 años (descenso del 20,4\%, casi un millón de niños menos que ahora). 
Menos niños y niñas, menos ‘adultos jóvenes'... y más mayores. En los próximos diez años habrá un millón y medio más de personas mayores de 65 años (un 17,6\% más que ahora). En realidad aumentarán todos los grupos de edad a partir de los 50 años (excepto el grupo de 80 a 84 años, que fueron generaciones más reducidas que nacieron con la Guerra Civil),

La esperanza de vida seguirá incrementándose, llegando a los 87 años en las mujeres y a los 81,8 años en los varones. Sigue siendo significativamente mayor en las mujeres, pero disminuyendo algo con respecto a los varones (aumenta 1,9 años, frente a los 2,5 años de los hombres). En el caso de la población de mayores de 65 años, la esperanza de vida llega a los 24,1 y 20,2 años respectivamente (un dato anecdótico: se duplicará las personas mayores de 100 años).

Pero aunque exista un aumento de la esperanza de vida, y a pesar de la disminución de la población, las defunciones aumentarán en los próximos diez años, debido al propio envejecimiento poblacional, estimándose que se producirán 4,1 millones de muertes (un $6,2 \%$ que los últimos diez años)

Se estima que en el 2017 tengamos un saldo vegetativo negativo, debido al descenso de la natalidad y al envejecimiento poblacional, es decir, que el número de fallecimientos supere al de nacimientos (hecho que no se ha producido en las últimas cuatro décadas).

Hemos analizado la situación al día de hoy y el envejecimiento de la población española previsto para los próximos diez años. Cabe preguntarse ahora qué ocurrirá más adelante, si esta tendencia se mantendrá. $Y$ todo parece indicar que, efectivamente, la estructura demográfica de nuestro país seguirá un continuo proceso de envejecimiento.

Veamos algunos datos de la Proyección de Población española a más largo plazo, a 40 años (INE, 2012). Como hecho más llamativo destacamos la disminución de la población (descenso de la natalidad y saldo migratorio negativo) y el progresivo envejecimiento de la misma. Así, si se mantienen las actuales tendencias demográficas, en el año 2.052 habrá un descenso de población, del 10\% con respecto a ahora.

Uno de los factores que incidirán en ello es el descenso de la natalidad (en los próximos cuarenta años nacerá un $20 \%$ menos que en las últimas cuatro décadas), a pesar de un previsible aumento de la fecundidad (aunque muy pequeño).

La esperanza de vida seguiría aumentando, llegando a los 90,7 años en las mujeres y 86,9 años en los varones, es decir, casi seis y ocho años más que en la actualidad (continuando la tendencia de aproximar la edad en los dos sexos).

Centrándonos en la población mayor de 65 años, la esperanza de vida será de 27,3 y 24 años respectivamente.

Una población más envejecida, que conllevará al aumento de los fallecimientos: en los próximos cuarenta años habrá 17,9 millones de defunciones, un $34 \%$ más que en los últimos 40 años. Más muertes que nacimientos, con el consiguiente crecimiento natural negativo.

Si analizamos la previsión por grupos de edad, la pérdida de población en el 2.052 afectará especialmente a las personas de entre 16 a 64 años (caerá un 32\%, casi 9,9 millones de personas menos) y a los menores de 15 años (disminución del 26\%, casi dos millones).

Por el contrario, las personas de mayor edad serán más: 7,2 millones de personas de más de 65 años (¡un aumento del 89\%!). Al principio de la segunda mitad del siglo XXI, más de un tercio de la población española superará los 65 años.

A la vista de lo anterior, está claro que 
- Habrá muchos más mayores. Tanto en números globales, en el número total, como especialmente en el porcentaje sobre el total de la población.

- Se vivirá más tiempo. Aumentará significativamente la esperanza de vida. Se vivirá con mejores condiciones de salud. Habrá más tiempo para, por ejemplo, formarse, asistir a clases...

\section{¿El envejecimiento de la población, un problema, o una oportunidad?}

Los datos anteriores son cifras, números objetivos. A partir de ellos se pueden extraer muchos análisis. Pero, por lo general, hablar de envejecimiento supone adoptar una visión negativa. En palabras de Pérez Salanova (2002: 21), "el envejecimiento de la población es considerado como un fenómeno de repercusiones negativas". Así, a raíz de esos datos, muchos realizan un planteamiento pesimista, catastrofista o insostenible.

Un ejemplo puede ser la tasa de dependencia o la relación entre las personas en edad de trabajar con respecto a las que no lo están (el cociente entre las personas menores de 16 años más las mayores de 64 y las personas entre 16 y 64 años) es actualmente del 51\%. La previsión es que se incremente un $9 \%$ en los próximos diez años $(59,2 \%)$, por lo que por cada diez personas en edad de trabajar, habrá seis 'inactivas' (menor de 15 o mayor de 64 años). Dentro de cuarenta años, por cada persona en edad de trabajar, prácticamente habría otra que no estaría en edad de hacerlo. Muchos sólo verán una población vieja, que no podrá mantenerse a sí misma. Una sociedad vieja, una sociedad 'anclada'. Envejecimiento en contraposición al desarrollo. Sin embargo, este hecho no coincide con la realidad, más bien al contrario.

Como dijimos en otra ocasión, la reproducción de los estereotipos negativos más frecuentemente asociados al envejecimiento (plasmada en las teorías de 'pandemia de las incapacidades': enfermedades, impotencia, decrepitud, disminución de capacidades mentales, fealdad, inutilidad, aislamiento, depresión, pobreza, etc.), supone un formidable obstáculo para elaborar un nuevo mapa conceptual de la valía, a veces inconmensurable, de la aportación de los mayores a su entorno familiar y social (Morón y García, 2007). Por ello, un alto nivel educativo supone una renta de autonomía importante a la hora de abordar los avances de la edad, de modificación de roles y estereotipos y de control de las filiares de exclusión.

Los mayores, las personas de edad avanzada, es un grupo más de la sociedad. Y tiene sus circunstancias específicas, como las tienen cualquier otro grupo poblacional. Hemos creado un estereotipo al relacionar a los mayores con 'problemas'. En parte viene de la concepción, o de la estructuración -ya típica- de la gestión de las edades en tres tiempos muy definidos, a saber, formación, empleo y jubilación. En los tiempos actuales, y mucho más en los venideros, no será esa la caracterización de un ciclo de vida.

Es necesario, pues, la adaptación de nuestra sociedad al aumento de la esperanza de vida, a la presencia de cada vez más mayores y en mejor estado. Y por ende, de poner en valor la capacidad de aprendizaje y adaptación de las personas de edad avanzada.

\section{El Aprendizaje a lo Largo de la Vida}

El punto de partida de la educación o formación de las personas más mayores se sitúa en el la propia Educación de Personas Adultas. Más concretamente, en la concepción del Aprendizaje a lo Largo de la Vida (LLL, Life Long Learning promovido por la UNESCO), 
definida como "toda actuación formativa que tiende a facilitar conocimientos y destrezas a las personas en edad postescolar, así como a promover en ellas actitudes y comportamientos valiosos orientados a propiciar su perfeccionamiento personal y profesional y la participación social" (Morón y Cruz, 2012).

Otra definición de referencia es la propuesta por la Comisión Europea (1995): El aprendizaje a lo largo de la vida es el desarrollo del potencial humano a través de un proceso sustentador continuo que estimula y faculta a los individuos para adquirir todos los conocimientos, valores, destrezas y comprensión que requieran a lo largo de toda su vida y aplicarlos con confianza, creatividad y gozo en todos los roles, circunstancias y entornos.

Es decir, una acción formativa, en cualquier momento del ciclo vital de una persona, que mejore sus conocimientos, destrezas, habilidades, competencias o cualificaciones, en cualquier ámbito personal, social o profesional.

Efectivamente, el término 'Educación a lo largo de la vida' fue acuñado y promovido por la UNESCO ya en la década de los setenta y ochenta del pasado siglo. Pero fue a raíz de posteriores documentos, principalmente de la Organización de Cooperación y Desarrollo Económico (OCDE, 1996) y el conocido informe Delors (1996), los que enfatizaron nuevamente la importancia del aprendizaje a lo largo de la vida, término y concepto que es común en la literatura actual.

Aprender a lo largo de toda la vida, abarca -por tanto-, a todas las edades; es una educación con un carácter integrador, en la búsqueda de conocimiento a través de la acción. La formación de las personas más mayores, y en general la educación de personas adultas, no es una 'repesca educativa', no es una segunda oportunidad, sino que es educación y formación propia e integral.

“El aprendizaje a lo largo de la vida es mucho más amplio que la provisión de una segunda oportunidad de educación e instrucción para personas adultas. Se basa en el punto de vista de que cada uno debe ser capaz, debe estar motivado, y activamente comprometido en aprender a lo largo de toda su vida. Este punto de vista del aprendizaje abarca el desarrollo personal y social de todo tipo y en cualquier escenario: formalmente, en las escuelas, en centros de formación profesional, terciaria o de adultos; e informalmente, en el hogar, en el trabajo y en la comunidad" (OECD, 1996).

Los principios educativos de esta concepción, del aprendizaje a lo largo de la vida, pasan por tres rasgos básicos:

Los principios educativos de esta concepción, del aprendizaje a lo largo de la vida, pasan por tres rasgos básicos:

a) En primer lugar, el carácter vitalicio de la educación, en base a:

- La educabilidad de la persona en cualquier etapa (desde el principio hasta el final), de la posibilidad de un proceso permanente de formación.

- La extensión vertical de la educación.

- La extensión articulada de la educación (integrada en la propia existencia humana).

b) Por otro lado, unidad y globalidad:

- En todos los niveles y en todas las modalidades educativas.

- La extensión horizontal: en multitud de situaciones y ámbitos (no solo en la escuela o institución formal). 
- Un nuevo estilo educativo: principalmente el Aprender a aprender y en el autoaprendizaje.

c) Por último, la universalidad, con la máxima del derecho a la Educación.

La educación debe producirse en cualquier momento, en cualquier lugar y con cualquier finalidad. Puede ser formal, informal o no-formal. $Y$ cubre todos los ámbitos posibles, sea social, personal, académico, profesional, técnico, etc. (longitudinal, vertical, multidimensional..., si se prefiere).

\section{Educación de Personas Adultas y Educación Permanente}

Ciertamente, el Aprendizaje a lo Largo de la Vida es el vocablo de moda en los últimos años, pero los pilares de este concepto vienen de más atrás. Educación de Personas Adultas, Educación Permanente... Como dice J.A. Fernández (2000, 24), "el término de educación permanente nació en el contexto de la educación de adultos, pero la finalidad del movimiento reformador de esos años era la transformación del conjunto del sistema educativo y de formación, a partir de la constatación de que las personas adultas necesitan seguir aprendiendo, aunque hayan ido a la escuela o a la universidad".

Los planteamientos actuales referentes a la formación o educación de personas adultas fijan objetivos principales:

- desarrollar una ciudadanía activa y comprometida;

- potenciar la educación en valores como eje del desarrollo humano en todas sus dimensiones, $y$

- responder a las exigencias de la Sociedad del Conocimiento.

Al día de hoy se mantienen vivas las orientaciones que recogía el Libro Blanco de Educación de Personas Adultas (MEC, 1986):

- Contemplar las cuatro áreas de conocimiento preferencia entre las personas adultas, es decir, que sea posible proporcionar una formación orientada al trabajo, una formación para el ejercicio de los derechos y responsabilidades cívicos, una formación para el desarrollo personal y una formación general o de base que constituirá el fundamento de todas las demás.

- Facilitar una educación integral.

- -Basar el diseño curricular en un sistema modular o en unidades formativas, con una organización, métodos y evaluación adaptadas.

- Conceder el principal protagonismo al proyecto de base territorial, sea una zona determinada, un barrio, un distrito, etc.

Así es, o debe ser, la educación de personas adultas y mayores, basada en los siguientes rasgos:

- Su carácter holístico y trans-sectorial: existe una extensa pluralidad de modalidades y métodos, en función de los objetivos y de las demandas de la población implicada. Un amplio universo formativo, con multitud de áreas, así como en la pluralidad de estructuras organizativas (presenciales, semipresenciales, a distancia).

- Ser una formación integral: Abarca y se centra en desarrollar todas las potencialidades de la persona, en aptitudes, valores, conocimientos, competencias, etc. 
- Teniendo en cuenta la amplia riqueza y variedad en los destinatarios.

- Con un carácter diferenciado, unas señas de identidad propias. Más que en cualquier otro tipo, aquí hay que tener en cuenta: la relevancia de los papeles sociales, su carácter voluntario, el papel de la experiencia, el protagonismo de los participantes, la responsabilidad y la autonomía de los educandos...

- Y defensora de la democratización educativa y cultural.

\section{En busca del 'envejecimiento activo'}

Los planteamientos actuales sobre los planes de acción hacia y con las personas más mayores se sustentan en los paradigmas del aprendizaje a los largo de toda la vida, de envejecimiento activo, de la calidad de vida, de las dimensiones de capacidad personal (autonomía, independencia).

La Organización Mundial de la Salud (OMS) dice que el envejecimiento activo es el proceso de optimización de oportunidades de salud, participación y seguridad con el objetivo de mejorar la calidad de vida a medida que las personas envejecen. Propiciar el envejecimiento activo es potenciar las capacidades de la persona cuando envejece, de llevar una vida productiva en la sociedad y en la economía.

No se es activo por estar bien de salud, por no tener dependencia, o por tener posibilidades económicas. Se es activo cuando se participa en las acciones sociales (en la cultura, economía, ocio...)

$\mathrm{Y}$, como decía, todos los planteamientos actuales sobre el envejecimiento, sobre el concepto de envejecimiento activo, coinciden en incluir el aprendizaje a lo largo de la vida.

Como señala el Libro Blanco sobre Envejecimiento Activo, se está revisando la amplitud y las nociones relacionadas con la propia idea de envejecimiento activo, sobre las que se sigue avanzando y matizando. Cabe hablar en este sentido de la transcendencia del concepto de ciudadanía, de la vivencia satisfactoria del propio proceso, de la importancia del aprendizaje a lo largo de la vida, de las relaciones intergeneracionales, de la capacidad de imaginación e innovación en su tratamiento, así como la importancia de la perspectiva de género (IMSERSO, 2011).

\section{Envejecimiento Activo y Educación a lo Largo de la Vida}

El citado Libro Blanco sobre el Envejecimiento Activo recoge una serie Conclusiones y Recomendaciones en el apartado referido a la 'Educación a lo Largo de la Vida', que por su actualidad y como publicación de referencia en este ámbito, merecen la pena reproducir, pues nos da una visión global sobre la cuestión (IMSERSO, 2011,316).

Así, aboga por la promoción de la Educación a lo largo de la vida sin limitación de edades con el fin de que la persona mejore sus competencias y que disponga de más oportunidades para optimizarlas y utilizarlas. Para ello habrá que contrarrestar los estereotipos negativos sobre las personas mayores, procurando no dar la imagen de que la educación a lo largo de la vida es algo inusual o extraordinario en edades avanzadas. Será necesario además:

- Mejorar la oferta actual de iniciativas de educación a lo largo de la vida existentes: procesos de enseñanza y aprendizaje, y modelos psicopedagógicos que fundamentan y orientan los programas que se ofertan. 
- Generar modelos de Buenas Prácticas en el ámbito de la educación a lo largo de la vida acordes con los modelos de valores sociales actuales y establecer criterios para la selección de materiales.

- Avanzar en el uso de las tecnologías de la comunicación por las personas mayores como herramienta facilitadora de nuevas oportunidades de participación social.

- Promover la estimulación y sensibilización de la sociedad, y especialmente de este colectivo, sobre la importancia de la educación a lo largo de la vida, procurando su implicación mediante foros, debate o cualquier otro medio.

- Promover foros y estructuras estables que permitan a todos los agentes implicados adultos mayores, profesionales y responsables de instituciones y políticos- trabajar de forma conjunta en la mejora de las oportunidades de educación a lo largo de la vida en su entorno.

- Desarrollar investigaciones que permitan conocer la oferta existente, la lógicapedagógica y organizativa subyacente en los diversos circuitos y los proceso de aprendizaje individuales y grupales.

- Mejorar los sistemas de formación de profesionales, orientándolos hacia competencias personales, relacionales y pedagógicas necesarias para liderar procesos de aprendizaje a lo largo de la vida para las personas mayores.

Desde una perspectiva más didáctica, a modo de propuestas metodológicas (con un enfoque 'cooperativo-constructivista'), se citan (IMSERSO, 2011, 295-296):

- Estimular procesos de reconstrucción a través de metodologías activas, prácticas, en entornos de aprendizajes reflexivos e interactivos.

- Proporcionar apoyos para que la influencia educativa entre docentes y educandos sea óptima, diseñando actualmente las tareas didácticas, procurando que los apoyos del profesorado evolucionen para potenciar roles cada vez más autónomos de los participantes y los grupos.

- Utilizar estrategias didácticas de carácter psicocéntrico, tales como:

- La investigación compartida, facilitadora del aprendizaje a partir de la experiencia personal y la reflexión en grupos de iguales.

- Los programas de educación intergeneracional, priorizando la calidad de la interacción y el desempeño de roles igualitarios, múltiples y cambiantes adaptativos- por parte de todos.

- El aprendizaje autodirigido que busca que la persona sea capaz de organizarse por sí misma y utilizar sus propios recursos: el uso de las TICS, de nuevas tecnologías de información y comunicación, bibliotecas, ofertas culturales, de experiencias enriquecedoras, como pueden ser viajes, relaciones...

- El aprendizaje basado en el estudio del caso, preferentemente elegido por los propios participantes, y sobre el que se formulen objetivos de aprendizaje y estrategias de abordaje.

- El desarrollo de proyectos definidos por el alumnado requieren que éstos se impliquen en todas su fases, tanto en la preparación (elección del tema, objetivos, elaboración del plan de acción, asignación de responsabilidades), su desarrollo e implementación (búsqueda de información, análisis, reuniones, desarrollo de los temas o tareas propuestas...), y su valoración o evaluación. 
Se han realizado otros Libros Blancos sobre las Personas Mayores o el Envejecimiento Activo en contexto autonómico, cuyas conclusiones y recomendaciones, en general, pueden ser extendibles a otros ámbitos. En el caso del de Andalucía, uno de los ejes se refería a 'Vivir en continua formación: La educación a lo largo de toda la vida'. Las conclusiones de este apartado tienen plena vigencia en cualquier territorio (Junta de Andalucía, 2010, 434-435):

- La educación a lo largo de la vida es tan importante para el envejecimiento activo que esta cambiando la imagen de las personas mayores.

- La educación a lo largo del ciclo vital debe ser abordada para y por las personas mayores.

- El catalogo de actividades educativas es un potente instrumento de formación permanente para el alumnado, pero debería ser ampliado y mejorado cualitativamente.

- La formación tiene una doble dirección: la persona mayor y la sociedad. Lo primero como crecimiento personal, lo segundo como difusor de experiencia, de la lectura, cultura, tradición y conocimiento.

- Destacamos la formación en las nuevas tecnologías y el aprendizaje de idiomas como acceso a la Sociedad del Conocimiento.

- Es fundamental en la educación a lo largo de la vida las relaciones intergeneracionales, que se deben propiciar en los diferentes programas de mayores.

- Debido a la importancia que tiene la jubilación, habría que incluir los Programas de Preparación a la Jubilación Activa, por los beneficios que tiene ésta en las personas jubiladas.

- La educación a lo largo de la vida debe ser accesible para todas las personas, independientemente del género, de su nivel económico y sociocultural.

- Los Programas Universitarios para Mayores (PUM) son un excelente instrumento para la educación a lo largo de la vida, pero deberían extenderse aun más a los contextos rurales.

- Para establecer las relaciones intergeneracionales es conveniente abrir los diferentes programas educativos a todas las edades.

- El intercambio cultural de las personas mayores de otras regiones, países y universidades es beneficioso para el envejecimiento activo.

- Es fundamental el actual cambio de rol del envejecimiento pasivo por el activo, al que están contribuyendo los diferentes programas innovadores en los que las personas mayores son los protagonistas como miembros activos de la sociedad.

- La investigación a lo largo del envejecimiento es importante como instrumento de análisis y evaluación de los diferentes programas de formación. Investigación que deberían realizar tanto las instituciones como las universidades, así como las personas mayores.

- Las mujeres deberían realizar, también, Programas de Preparación a la Jubilación Activa (PPJA), independientemente que no hayan trabajado de forma remunerada.

- Seria muy conveniente, de acuerdo con la evolución de la población, que se potenciaran grupos de autoformación impartida por las personas mayores. 
- Es necesario crear un material transversal para formar a los niños y niñas en el conocimiento de proceso de envejecimiento activo, como miembros de una sociedad envejeciente y envejecida.

\section{Personas Mayores y Universidad}

Las persones mayores no son un lastre de la sociedad. Ahora que ya sabemos mucho de los mayores, que se comprueba el envejecimiento de la población, ya es hora de buscar soluciones, de llevar a cabo propuestas... de descubrir las potencialidades de las personas mayores. $Y$ entre estas potencialidades, una, sin duda -y muy importante-, es la formación. Una formación distinta a la de un niño, a la del adolescente, a la del joven universitario, a la del adulto que busca una especialización laboral... pero una formación.

La formación del profesorado (de formadores, del educador, si se prefiere) de la personas mayores es, ante todo, la formación de un formador o formadora (de un educador o educadora). Algunas estrategias, técnicas, metodologías... serán específicos, para trabajar con las personas de edad avanzada (a igual que sucede con un formador de educación infantil, de primaria o de universidad). Pero también hay muchos elementos coincidentes. En mi opinión personal, hay mucha más coincidencia, más peso en las similitudes, entre el formador de mayores y cualquier otro formador, que en la especialidad o las diferencias específicas entre ambos. De hecho, por ejemplo, también hay personas de avanzada edad en las aulas universitarias, en distintos Grados, en Master, Doctorado... y conviven con otros participantes de edades inferiores. $Y$ no por eso el profesor o profesora de turno es distinto a otro compañeros que sólo tenga a educandos más 'jóvenes'. Eso puede comprobarse en algunos estudios que se han centrado en el alumnado de mayor edad.

Valga como ejemplo el estudio de Leal, Toribio y Sotomayor (2009) sobre el alumnado universitario de avanzada edad, con sus características, motivaciones, valores, obstáculos y otras variables de interés que nos ayudan a conocer esta realidad. A título informativo, reseñar la afirmación final recogida en las conclusiones de los autores: "Los resultados obtenidos nos han puesto de manifiesto una serie de características, situaciones y diferencias que van a afectar a la iniciativa de emprender unos estudios universitarios. De esta manera, todas ellas han de ser consideradas a la hora de llevar a cabo una oferta formativa dirigida a dicha población, para tener en cuenta las particularidades de su demanda y de esta forma alcanzar una mayor participación de dicha oferta a esta última, logrando así facilitar su acceso a la universidad" (p. 155).

Siguiendo a Moreno (2009), históricamente, no podemos decir que entre los objetivos prioritarios de la Universidad se sitúen las personas mayores. Tampoco al revés. Pero ya en el siglo XVIII encontramos algunas experiencias de mayores en la universidad, si bien hay que esperar a la segunda mitad del siglo pasado para encontrar ejemplos más significativos y numerosos.

Actividades culturales universitarias, ciclos de conferencia, universidades de la Tercera Edad (en España la primera Aula de la Tercera Edad se creó en 1978), enseñanzas 'formales' para los más mayores (la Universidad de Quebec fue la pionera en ofertar un Certificado de Primer Ciclo para estudiantes mayores de 55 años)... han ido creando una amplia red, que en nuestro país llega prácticamente a todas las Universidades.

La expansión en la última década del siglo pasado y en la primera del presente ha sido muy importante. Existen además múltiples modalidades que conviven entre sí (Asociaciones y 
Agrupaciones de difusión cultural o formación permanente; centros o instituciones de Extensión Universitaria; Programas Universitarios para Alumnos y Alumnas Mayores).

Poco a poco surge el interés en dotar a los programas universitarios para los mayores con una serie de características:

- La función social de los programas,

- La organización de los contenidos de cara a una mejor inserción social del alumnado,

- El diseño de un curriculum adaptado y coherente,

- Dar respaldo a la investigación donde las personas mayores fueran parte activa y pasiva de la misma (Moreno, 2009: 21).

Cada vez existe un mayor número de experiencias de mayores en la universidad, tanto en el ámbito de la metodología, investigaciones... (Orte, 2006; Lemieux, 1997; Cabedo y Alfageme, 2006, Velazquez, 2006; Velázquez y Fernández, 1998, Velázquez et alt, 2000; Zorita, Yuste y Gázquez, 2006, etc.). También caben destacar los materiales emanados de los Encuentros Nacionales de Programas Universitarios para Mayores, que se han celebrado en distintas Universidades, además de recomendar otras obras específicas sobre Educación y Personas Mayores: (Bedmar, Fresneda y Muñoz, 2004; Colom y Orte, 2001; Escarbajal, 2004; García, 2004; García y Bedmar, 2002; García y Sánchez, 1998; Sáez, 2002; Sáez, 2003; Yuni y Urbano, 2005).

La educación de los más mayores también "se convierte en una oportunidad interesante para generar procesos de reflexión y aprendizajes, que nos permiten avanzar en la formación didáctica del profesorado de dichos programas. Pensamos además que estos procesos de formación generan traspasos, interacciones y puentes entre la docencia que estos profesores desarrollan en la Universidad convencional y la Universidad de Mayores. Este reto, por tanto, puede constituirse en una oportunidad para generar innovación educativa entre el profesorado de la universidad" (Lirio y Morales, 2012, 155).

En definitiva, y como corolario final, el binomio Educación-Personas Mayores debe ser una realidad, dos ámbitos que guarden una estrecha relación. En una sociedad en progresivo envejecimiento, como es la nuestra, abre una oportunidad, un nicho en la que la formación del profesorado (la formación de los educadores y formadores) tiene un campo de acción muy importante y en crecimiento. Eso sí, una formación con un carácter específico dentro de la Educación Permanente y de la Educación de Personas Adultas, que requiere un enfoque diferente tanto en el planteamiento conceptual como en el procedimental o metodológico.

$Y$ todos los estamentos debemos implicarnos en potenciar esta realidad: "Habrá que dotarse de los medios de desarrollar el apetito de la Educación y la Formación a lo largo de la Vida, y abrir y generalizar permanentemente el acceso a nuevas formas de conocimiento" (Comisión Europea, 1995, 2). Hay que aprovecharse de la voluntariedad de los más mayores, del 'ocio educativo', que vuelvan a dar ganas de formarse a los que no han querido o no han podido integrarse en los sistemas clásicos de enseñanza.

\section{Bibliografía}

Bedmar, M., Fresneda, M.D. y Muñoz, J. (2004). Gerontagogía. Educación en personas mayores. Granada, Universidad de Granada. 
Cabedo Manuel, S. y Alfageme Chao, S. (dir.) (2006). Los programas universitarios para mayores en España: Una investigación sociológica. Castellón, Universidad Jaime I.

Colom Cañellas, A.J. y Orte Socías, C. (2001). Gerontología educativa y social. Pedagogía Social y personas mayores. Palma de Mallorca, Universidad de las Islas Baleares.

Comisión Europea (1995). Libro Blanco sobre la educación y la formación. Enseñar y aprender. Hacia una sociedad cognitiva. Bruselas, Oficina de Publicaciones Oficiales de las Comunidades Europeas.

Delors, J. et alt. (1996). La educación encierra un tesoro. Informe a la UNESCO de la Comisión Internacional sobre la Educación para el siglo XXI. Madrid, UnescoSantillana.

Escarbajal de Haro, A. (2004). Personas mayores. Educación y emancipación. La importancia del trabajo cualitativo. Madrid, Dykinson.

Fernández, J.A. (2000). El redescubrimiento de la Educación Permanente. Educación XXI, 3, 21-51.

García Mínguez, J. (2004): La educación de personas mayores. Ensayo de nuevos caminos. Madrid, Narcea.

García Mínguez, J. y Bedmar, M. (coords.) (2002). Hacia la educación intergeneracional. Madrid, Dykinson.

García Mínguez, J. y Sánchez García, A. (1998). Un modelo de educación en los mayores: la interactividad. Madrid, Dykinson.

IMSERSO (2011). Libro Blanco sobre el Envejecimiento Activo. Madrid, IMSERSO-Ministerio de Sanidad, Política Social e Igualdad.

INE (2013). Proyección de la Población a Corto Plazo en España. Madrid, Instituto Nacional de Estadística.

INE (2012). Proyección de la Población a Largo Plazo en España. Madrid, Instituto Nacional de Estadística.

Junta de Andalucía (2010). Libro Blanco del envejecimiento activo. Sevilla, Consejería para la Igualdad y Bienestar Social.

Leal Jiménez, A., Toribio Muñoz, M.R. y Sotomayor González, S. (2009). Nuevos públicos para una Universidad próxima: los usuarios mayores de 40 años. Cádiz, Dirección General de Universidades, Junta de Andalucía.

Lemieux, A. (1997). Los programas universitarios para mayores: Enseñanza e investigación. Madrid, IMSERSO-Ministerio de Trabajo y Asuntos Sociales.

Lirio Castro, J. y Morales Calvo, S. (2012). El reto de la formación del profesorado para una enseñanza e calidad en las universidades de mayores. Revista Interuniversitaria de Pedagogía Social. 19, 155-166.

MEC (1986). Libro Blanco de Educación de Personas Adultas. Madrid, Ministerio de Educación y Ciencia.

Moreno Tello, S. (2009). Las Aulas universitarias de Mayores en Andalucía: Un estudio sectorial. Cádiz, Dirección General de Universidades, Junta de Andalucía. 
Morón Marchena, J.A. y García Martínez, A. (2007). Exclusión e Intervención socioeducativa con mayores. III Jornadas Pedagógicas y de Educación Social 'Educación, Marginación y Exclusión Social'. Huelva, Universidad de Huelva.

Morón Marchena, J.A.; Cruz Díaz, M.R. et alt. (2012). Las Universidades Populares: Bases Conceptuales. Madrid, Federación Española de Universidades Populares-FEUP.

OECD (1996). Making Lifelong Learning a Reality for All. Paris, OECD.

Orte Socías, C. (Coord.) (2006). El aprendizaje a lo largo de toda la vida. Los programas universitarios de mayores. Madrid, Dykinson.

Pérez Salanova, M. (2002). La participación de las Personas Mayores. Apuntes para una agencia de intervenciones gerontológicas. Revista Interuniverstaria de Formación del Profesorado , 45, 21-32.

Sáez Carrera, J. (coord.) (2002). Pedagogía social y programas intergeneracionales: educación de personas mayores. Archidona (Málaga), Aljibe.

Sáez Carreras, J. (coord.) (2003). Educación y aprendizaje en las personas mayores. Madrid, Dykinson.

Velázquez, M. (2006). Reflexiones sobre los PUM. Universidad de Sevilla. Secretariado de Publicaciones.

Velázquez, M. y Fernández, C. (1998). Las Universidades de Mayores. Una aventura hecha realidad. Estimulación y desarrollo en el último gramo del ciclo vital. Sevilla, Universidad de Sevilla.

Velázquez, M., Fernández, C.; Holgado, M.A., Guirao, M. y Sánchez, M. (2000). Guía de Programas Universitarios de Personas Mayores. Madrid, Ministerio de Trabajo y Asuntos Sociales-IMSERSO.

Yuni, J.A. y Urbano, C.A. (2005). Educación de adultos mayores. Teoría, investigación e intervenciones. Córdoba, Brujas.

Zorita, C., Yuste, N. y Gázquez, J.J. (2006). Mayores en la Universidad: Derecho, necesidad satisfacción. Almería, Universidad de Almería. 


\section{Autores}

Juan Agustín Morón Marchena

Dr. en Pedagogía, es Profesor Titular de la Universidad Pablo de Olavide de Sevilla (Área de Teoría e Historia de la Educación) y Director del Departamento de Educación y Psicología Social. Anteriormente ha sido profesor de las Universidades de Huelva y de Sevilla. Sus principales líneas de investigación son: Educación para la Salud, Educación para el Desarrollo, Docentes, Educación de Personas Mayores-Envejecimiento Activo. Coordina y participa en diversos proyectos de investigación sobre dichas temáticas. Miembro del Grupo de Investigación de Educación de la Universidad Pablo de Olavide (GEDUPO) 